\title{
THE PATHOLOGY OF PULMONARY HEART DISEASE
}

\author{
BY \\ FLORENCE MCKEOWN \\ From the Institute of Pathology, Belfast \\ Received July 12, 1951
}

Right ventricular hypertrophy has been described in association with a variety of pulmonary disorders. In some of these there is an obvious relationship between the changes in the right ventricle and the severity of the pathological lesions in the pulmonary tissue, but in others it is difficult to find any correlation. The following review of the incidence of pulmonary heart disease in routine post-mortem material attempts to estimate the importance of some of the lesions of the pulmonary vascular tree as a morphological basis for the cardiac hypertrophy. Of 6770 necropsies available for study, 111 showed hypertrophy of the right ventricle secondary to disease of the lungs. In these cases any complicating factor such as essential hypertension, valvular lesions, or coronary vascular disease was excluded. The thickness of the ventricular wall was accepted as the index of hypertrophy, and while the ventricle was $5 \mathrm{~mm}$. or more in thickness in all the cases studied, it was much more than this in the great majority.

The cases are classified according to their ætiology in the Table, where it is seen that in the material in this department emphysema is the most common cause. It is not intended to discuss all the various conditions listed, as similar analyses have been presented by Brenner (1935), Griggs et al. (1939), Scott and Garvin (1941), and Spain and Handler (1946). There are, however, certain subgroups that are of some interest because the absence of morphological lesions in the lung parenchyma suggests that the cause of the increased blood pressure is to be sought in the vascular tree itself. Such cases fall naturally into three groups.

Group I comprises those cases where there is an obvious obstructive lesion of the pulmonary vascular system to account for the changes in the heart, namely four of pulmonary arteritis, two of tumour embolism, and five of chronic pulmonary embolism.

Group II, the direct antithesis of Group I, consists of four cases where pulmonary hypertension or right ventricular hypertrophy occurs in the absence of any demonstrable histological lesion in the pulmonary vascular system. These constitute the "unknown cause" group or so-called primary pulmonary hypertension.

Group III includes 101 cases of emphysema, 39 with, and 62 without right heart ventricular hypertrophy. An additional 163 normal controls are included, to provide a study of the age changes within the pulmonary vascular system, and so to establish a standard of comparison for the influence of pulmonary disease in the genesis of sclerosis of the pulmonary vessels.

\section{Group I. With Obstructive Pulmonary Vascular Lesion}

Pulmonary Arteritis. Four cases of arteritis were found in this series. The ages of the patients concerned were $10,13,24$, and 42 years. Three presented clinically with right heart failure of several months' duration and of obscure origin. Post mortem, an unexplained hypertrophy of the right ventricle was found, but on histological examination the large, medium, and small pulmonary vessels were the seat of an acute and healing arteritis, showing thrombosis, medial necrosis, and inflammatory infiltration going on to recanalization, scarring of the media and adventitial fibrosis (Fig. 1). The segmental scars in the media, from which elastic tissue was entirely absent, were very striking and such scars are regarded as characteristic of the healed stage of polyarteritis (Fig. 2). The pathogenesis of these cases is uncertain. On histological grounds alone the picture is identical with 
TABLE

Distribution of InCiDENCE of Right Ventricular HyPertrophy in 6770 NeCropsies

\begin{tabular}{|c|c|c|c|}
\hline & \multirow{2}{*}{ Incidence } & \multicolumn{2}{|c|}{ Cause of Death } \\
\hline & & $\begin{array}{l}\text { Right heart } \\
\text { failure }\end{array}$ & Other \\
\hline 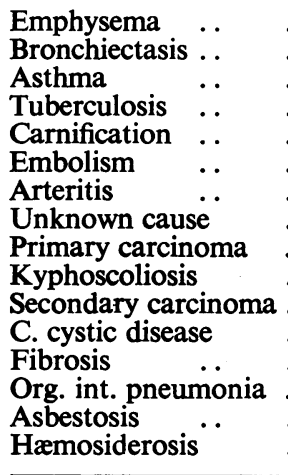 & $\begin{array}{c}39 / 101 \\
17 / 80 \\
14 / 24 \\
7 / 92 \\
5 / 42 \\
5 / 103 \\
4 \\
4 \\
3 \\
3 \\
2 \\
2 \\
2 \\
2 \\
1 \\
1\end{array}$ & $\begin{array}{r}20 \\
6 \\
9 \\
3 \\
3 \\
4 \\
4 \\
2 \\
2 \\
1 \\
1 \\
1 \\
1\end{array}$ & $\begin{array}{r}19 \\
11 \\
5 \\
4 \\
2 \\
1 \\
2 \\
3 \\
1 \\
1 \\
1 \\
2 \\
1 \\
1 \\
-\end{array}$ \\
\hline Total .. & 111 & 57 & 54 \\
\hline
\end{tabular}

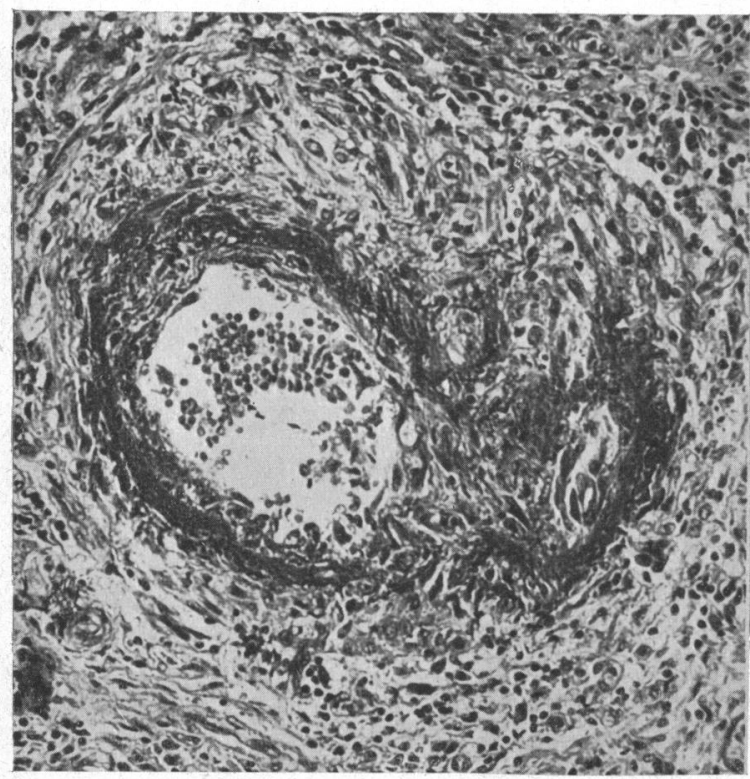

Fig. 1.-Pulmonary arteritis: vessel showing medial necrosis, and perivascular inflammatory infiltration (H.E., $\times 180)$.

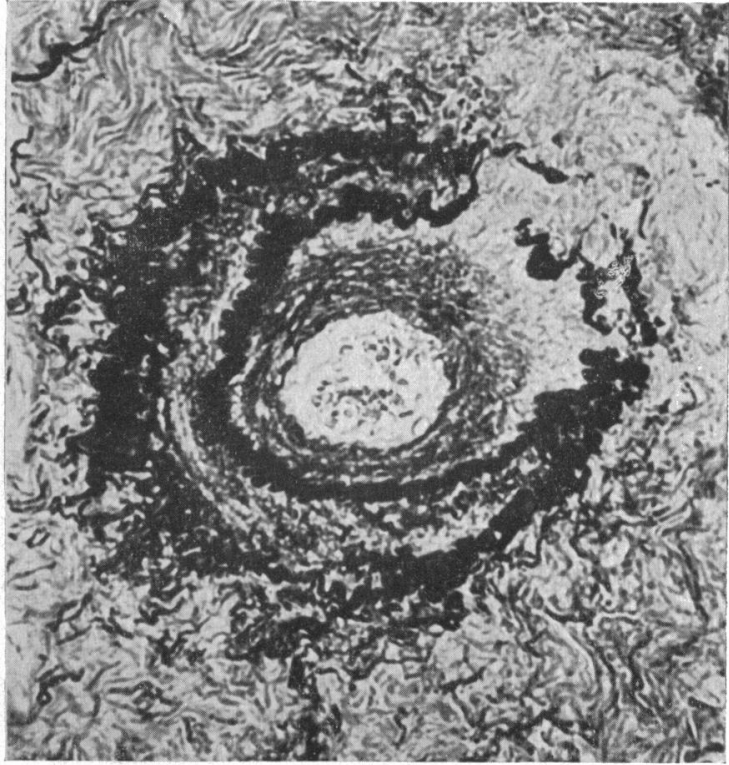

FIG. 2.- Healed arteritis: pulmonary artery showing intimal fibrosis and elastic tissue defect in media (H.V.G., $\times 160)$. 
polyarteritis nodosa when pulmonary involvement is only part of a widespread arteritis. It is difficult, however, to explain the localization of the lesion to the lungs, though some cases of polyarteritis nodosa do show an unusual restriction to certain sites. It may be that the lungs act as the " shock" organ, or that the nature of the allergen, its route of entry into the circulation, or the character of the " soil," or indeed all those factors that influence any hypersensitive reaction, modify the process so that only the pulmonary vascular system suffers.

The other alternative is that these cases of arteritis are examples of primary pulmonary hypertension, with secondary vascular necrosis, and that the condition is the counterpart of malignant hypertension in the greater circulation. The vascular changes, however, are not entirely comparable with those found in malignant hypertension, since all sizes of vessel are involved, intense inflammatory infiltration is a feature of the acute phase, the lesions are essentially segmental in character, and show a tendency to undergo healing. Furthermore, in this material vascular necrosis in the lungs in the

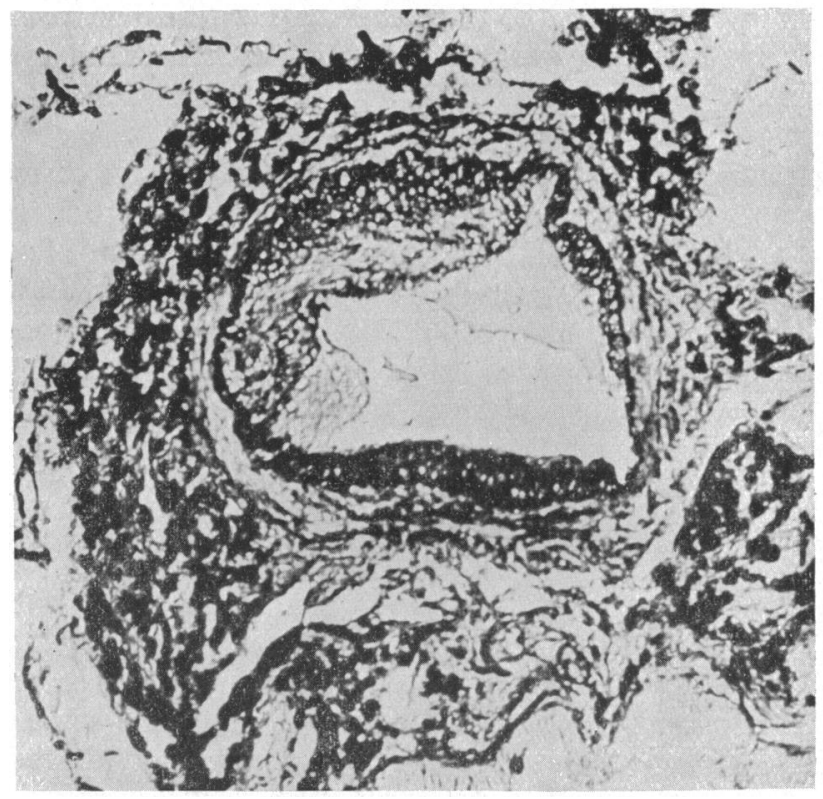

Fig. 3.-Pulmonary embolism: vessel showing organized thrombus, with reconstitution of the lumen and elastification of the intimal plaque (H.V.G., $\times 150)$.

presence of severe secondary pulmonary hypertension has not been noted; though Parker and Weiss (1936) describe necrotizing pulmonary arteriolitis in five cases of advanced mitral stenosislesions that resemble closely the vascular changes found in the kidneys in malignant hypertension, but not those described in these cases of arteritis. Appreciably higher pulmonary pressures have been recorded in cases of Eisenmenger's complex than in mitral stenosis, without the occurrence of vascular necrosis, but in this condition the adaptation of the pulmonary vasculature to abnormal pressure from birth and the development of marked vascular sclerosis probably protect the bloodvessels from the effects of extreme hypertension. One of the few references to pulmonary arteritis is in association with a case of Eisenmenger's complex described by Old and Russell (1950). The evidence available, therefore, tends to support the concept of a primary pulmonary arteritis rather than a vascular necrosis secondary to essential malignant pulmonary hypertension. Whatever the ætiology, the illustrations accompanying cases reported as examples of primary pulmonary vascular sclerosis make it apparent that some of them at least belong to this group. 
Tumour Embolism. Two cases of tumour embolism with right heart hypertrophy were encountered. In one, symptoms of embarrassment of the right ventricle had been present for two years, the patient dying from right heart failure five years after removal of a uterine chorionepithelioma. Post mortem, most of the large pulmonary vessels were filled with thrombus in which tumour cells were actively growing and invading their walls and then apparently undergoing arrest of growth, leaving vessels with medial scars devoid of elastic tissue, not unlike those seen in primary arteritis. Most cases of tumour embolism conform to the clinical picture of subacute cor pulmonale as described by Greenspan (1934) and Brill and Robertson (1937)-a right-sided hypertrophy occurring in association with diffuse carcinomatosis of the lungs, developing during the last few weeks of life. The unusual feature of this case, therefore, is the prolonged clinical course which is probably explained by the peculiar characteristic of the tumour in undergoing spontaneous regression after periods of active growth within the pulmonary vascular system.

The other case illustrates the second mechanism whereby tumour metastases may embarrass the right heart. A primary carcinoma of the œsophagus with extensive involvement of the pulmonary perivascular lymphatics had evoked a fibrous tissue reaction around the blood-vessels with secondary occlusion of their lumina. This type of secondary endolymphatic carcinomatosis is the commonest cause of subacute cor pulmonale.

Chronic Pulmonary Embolism. The present analysis revealed five cases of chronic pulmonary embolism with right ventricular hypertrophy, four of these dying from right heart failure. The duration of symptoms varied from a period of months to five years. Such a prolonged course, however, is not unique, since in a case mentioned by Castlemann and Bland (1946) there was a nine years' history of heart failure. One case showed almost complete and long-standing occlusion of both main pulmonary arteries and their branches, serving to illustrate the great reserve power of the pulmonary vascular bed. When recurrent pulmonary embolism has been present for some time it may present certain difficulties in histological interpretation, for the organization of emboli in the vessels leads to the formation of intimal thickenings which undergo elastification and are almost identical with those found in pulmonary arteriosclerosis (Fig. 3). This has been pointed out by Duguid (1946) who suggested that intimal plaques in the coronary vessels and aorta may be the result of organized thrombi and may be indistinguishable from arteriosclerosis. Harrison (1948) in a study of experimentally produced embolism traced the development of a fibro-elastic intimal plaque with reconstitution of the lumen in vessels the seat of previous embolism. Saphir (1947), too, has shown that even tumour emboli may undergo the same fate. Since, therefore, minor degrees of chronic pulmonary embolism are not uncommon, and fibro-elastic intimal plaques can evolve on this basis, care must be taken in the interpretation of sclerotic lesions of the pulmonary vessels. Some of the recorded cases of so-called primary pulmonary vascular sclerosis also may be explained in this way.

\section{Group II. Primary Pulmonary Hypertension}

In the second group of cases there were four in which right ventricular hypertrophy occurred in the absence of any morphological lesion that could be demonstrated in the pulmonary vasculature or parenchyma. These are regarded as examples of primary pulmonary hypertension. There is some confusion as to what constitutes primary pulmonary hypertension. It is attributed by Gilmour and Evans (1946) to a variety of pathological processes in the pulmonary vascular tree in the absence of any known cardiac or pulmonary disorder or any extrinsic disease capable of damaging the pulmonary vascular system: by certain standards this might be considered as a variety of secondary pulmonary hypertension. On the other hand, East (1940) suggests that in idiopathic or essential pulmonary hypertension there is little or no vascular change to which the right ventricular hypertrophy and failure may be attributed. The cases under consideration conform to this classification. De Navasquez (1940) discusses the condition as one of right ventricular hypertrophy of unknown origin, and presents three cases similar to those in this series: he questions whether the hypertrophy of the right ventricle justifies the opinion that it is due to increased work and 


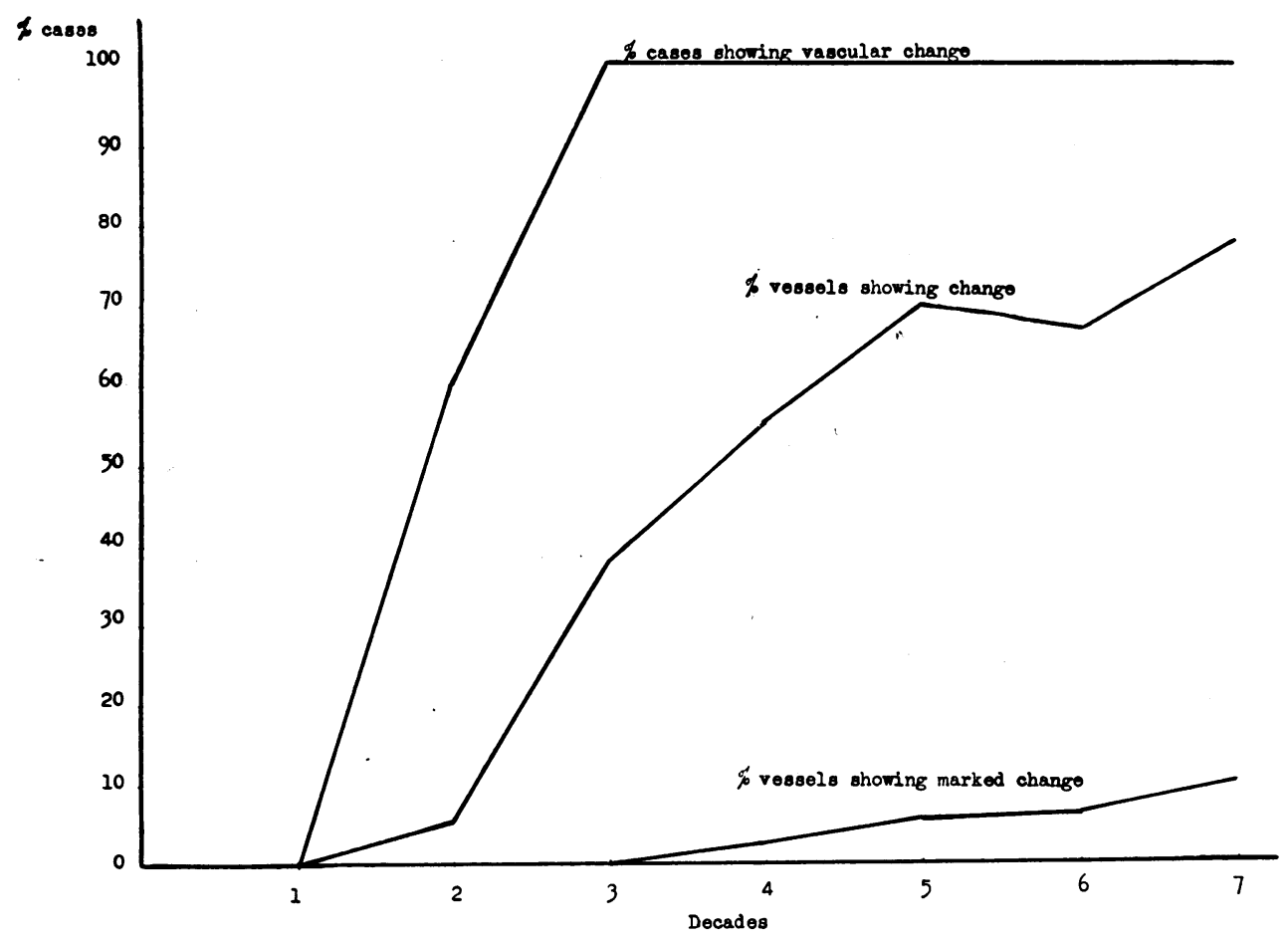

Fig. 4.-Graph showing incidence and extent of vascular sclerosis in control group.

analogous to left ventricular hypertrophy in systemic hypertension; and since no comparable lesion is found in the pulmonary blood-vessels he believes the existence of pulmonary hypertension is conjectural, and that idiopathic right ventricular hypertrophy would be a better name. It is well known, however, that changes in the systemic vessels are not invariably present in hypertension and are generally regarded as secondary to the hypertension. Since, also, the structure of the pulmonary arterioles differs from that of the systemic vessels, the failure to show hyaline arteriolosclerosis does not indicate that hypertension is not present. The condition may be analogous to systemic hypertension and perhaps initiated by a humoral or neurogenic mechanism. That such exists in the experimental animal has been shown by Daly et al. (1947) who demonstrated a vasomotor mechanism in dogs capable of causing a rise in pulmonary blood pressure. As it appears, therefore, from a study of this group of cases, that right ventricular hypertrophy can occur in the absence of any morphological change in the pulmonary blood-vessels, it is evident that too facile a correlation between pulmonary vascular lesions and changes in the right heart should be avoided.

\section{GROUP III. EMPHYSEMA}

There were 39 cases of emphysema with right ventricular hypertrophy, 20 dying of right heart failure. The importance of emphysema in the production of pulmonary vascular sclerosis is disputed, and sometimes an attempt is made to correlate the degree of vascular change with the presence or absence of right ventricular hypertrophy. Karsner (1933) considered emphysema to be the most important pulmonary condition in the genesis of arteriosclerosis of the pulmonary vascular system. Parker (1940), however, found pulmonary vascular changes in 80 per cent of 32 cases of emphysema, but concluded that there was no causal relationship between this change and the hypertrophy of the right ventricle. Evans (1951) has suggested that in conditions such as emphysema which may produce a slight rise in pulmonary pressure, hypoplastic changes in the vascular media under the 


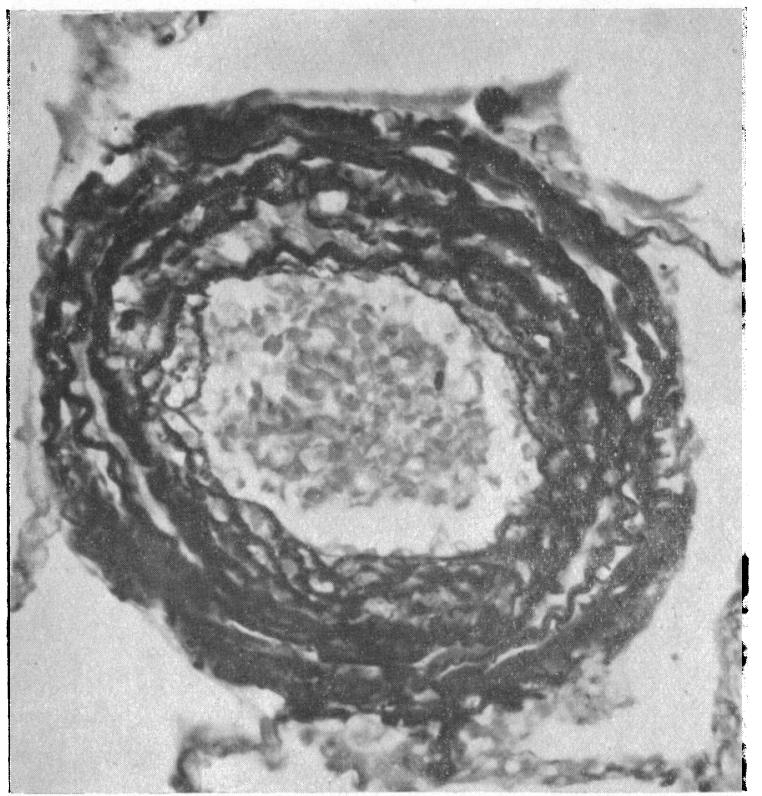

FIG. 5.-Control group: vessel showing marked intimal musculo-elastic hyperplasia (H.V.G., $\times 406$ ).

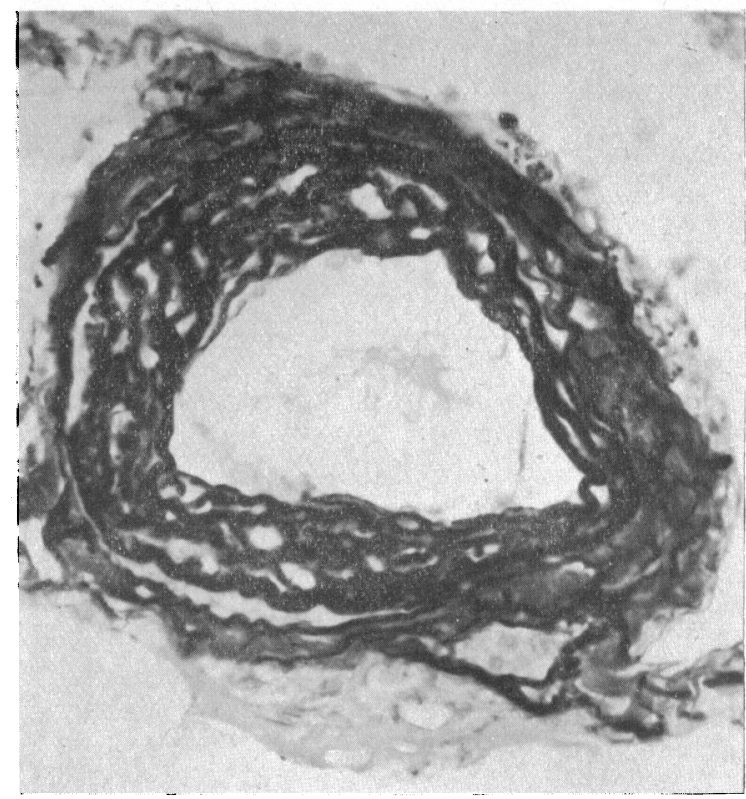

Fig. 6.-Emphysema: vessel showing musculo-elastic thickening of the intima (H.V.G., $\times 406)$.

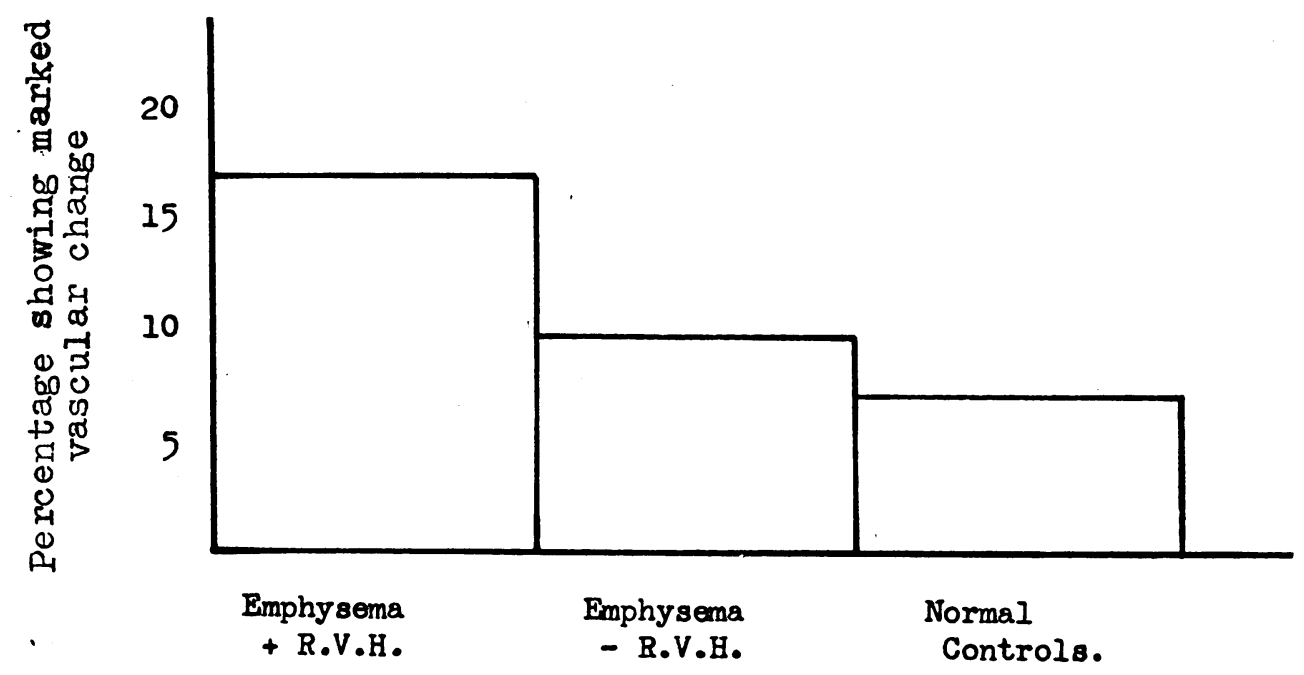

FIG. 7.-Shows incidence of marked pulmonary vascular sclerosis in emphysema compared with normal controls of similar age distribution.

stress of mild hypertension might lead to excessive compensatory endarteritis and eventually result in serious pulmonary hypertension. Before the significance of vascular changes in the lungs in emphysema can be correctly assessed it is essential to establish in control material the frequency and extent of sclerosis of the pulmonary vessels at all ages, but especially in the later decades. Only in this way can standards of normality be created against which the influence of conditions like emphysema can be compared. Brenner (1935) found that sclerosis was present in 97 per cent of 
100 consecutive necropsies. Welch and Kinney (1948) described some degree of arteriosclerosis in every case from the fourth decade on. Civin and Edwards (1951) studied the changes in the different decades, and found them constantly present, but only exceptionally were they of such a degree as to narrow the lumen to any appreciable extent. Similar studies have been carried out in this department by Dr. R. A. Neely, in an analysis of 63 cases representative of seven decades, and carefully selected to exclude those with chronic disease of the heart or lungs. The changes found included varying degrees of intimal thickening due to fibro-elastic or musculo-elastic intimal hyperplasia, and affected all sizes of vessels to some extent. As representative of the whole group the incidence and severity of the lesions in the small arteries are presented in Fig. 4, and it is seen that in the later decades sclerosis is consistently present, and that in a small percentage of cases the degree of vascular change is marked. An example of such sclerosis is shown in Fig. 5, and can be compared with Fig. 6, which shows a similar degree of intimal hyperplasia in a case of emphysema. The incidence of vascular change in a further 100 control cases selected from the fourth to the seventh decade was compared with its frequency in the 39 cases of emphysema with, and in 62 cases of emphysema without right ventricular hypertrophy. The results are seen in Fig. 7. Taken as a whole, the incidence of severe sclerosis in these three groups shows little significant difference, 7 per cent in controls, 10 per cent in emphysema without right ventricular hypertrophy, and 17 per cent in emphysema with enlargement of the right ventricle. Since such a degree of vascular change occurs in controls without hypertrophy of the right ventricle and is absent in 83 per cent of cases of emphysema with right ventricular hypertrophy it cannot be regarded as a morphological basis for the hypertension. The most that can be concluded from this study is that emphysema accentuates slightly the normal age changes found in the pulmonary circulation. The reason, therefore, why some patients with emphysema develop right ventricular hypertrophy is not to be found in a study of the changes in the small vessels. The whole problem of right heart hypertrophy in emphysema is complex, and from the histological standpoint there is no constant pathological change found that might be regarded as a morphological basis for its development. One important factor is probably the reduction of the pulmonary reserve by gradual obliteration of the capillary bed. It is suggested by Spain and Handler (1946) that the resistance to flow is not necessarily based solely on the anatomical obliteration of the vascular bed, but in addition there is a functional pressure resistance relation occurring in functioning pulmonary tissue as exerted over a long period. Other factors of doubtful significance are diffuse fibrosis, polycythæmia, overfilling of the heart, increased cardiac output, and the existence of vascular shunts. In view of the different pressure relationships in the pulmonary and bronchial circulation, and the relatively small size of the bronchial arteries it is difficult to imagine how vascular shunts could play an important part in the prevention or production of pulmonary hypertension. It has been suggested that anoxia is an important factor in raising the pulmonary blood pressure, but conflicting results have been obtained in emphysematous patients in attempts to find a correlation between the arterial pulmonary pressure and the degree of arterial oxygen saturation.

Whatever the factors concerned, it is only the purpose of this paper to stress the almost constant occurrence of some degree of pulmonary vascular sclerosis in normal control material in the later decades. This observation should be borne in mind not only in the interpretation of vascular lesions in association with emphysema, but in any consideration of a possible morphological basis for pulmonary hypertension.

\section{SUMMARY}

An analysis is presented of the incidence in 6770 necropsies of right ventricular hypertrophy in association with pulmonary disease. Three groups of cases are discussed in some detail. Group I comprises those cases where there is an obvious obstructive lesion of the pulmonary vascular system namely arteritis, tumour embolism, and chronic pulmonary embolism.

Group II consists of four cases of so-called primary pulmonary hypertension, where hypertrophy of the right ventricle occurs in the absence of any demonstrable lesion in the pulmonary vasculature or parenchyma. 
Group III includes all the cases of emphysema, with or without right ventricular hypertrophy.

Though attempts have been made to correlate the right ventricular hypertrophy with vascular lesions in the lungs, it is shown that such lesions as are found in the small vessels conform to the normal age changes of the pulmonary vascular system, and therefore cannot be regarded as the morphological basis for the enlargement of the right heart.

\section{REFERENCES}

Brenner, O. (1935). Arch. intern. Med., 56, 211.

Brill, I. C., and Robertson, T. D. (1937), Arch. intern. Med., 60, 1043.

Castlemann, B., and Bland, E. F. (1946). Arch. Path., 42, 581.

Civin, W. H., and Edwards, J. E. (1951). Arch. Path., 51, 192.

Daly, I. de B., Dake, H., and Weatherall, J. (1947). Seventeenth International Physiological Congress, Abstracts of Communications, Oxford, p. 12.

De Navasquez, S. (1940). Brit. Heart J., $2,177$.

Duguid, J. B. (1946). J. Path. Bact. 58, 207.

East, T. (1940). Brit. Heart J., $2,189$.

Evans, W. (1951). Brit. med. J., 1, 582.

Gilmour, J. R., and Evans, W. (1946). J. Path. Bact., 58, 687.

Greenspan, E. B. (1934). Arch. intern. Med., 54, 625.

Griggs, D. E., Coggin, C. B., and Evans, N. (1939). Amer. Heart J., 17, 681.

Harrison, C. V. (1948). J. Path. Bact., 60, 289.

Karsner, H. T. (1933). Sclerosis of the Pulmonary Artery in Cowdry, E. V. (1933). Arteriosclerosis. New York, Macmillan.

Old, J. W., and Russell, W. O. (1950). Amer. J. Path., 26, 789.

Parker, F., and Weiss, S. (1936). Amer. J. Path., 12, 595.

Parker, R. L. (1940). Ann. intern. Med., 14, 795.

Saphir, O. (1947). Amer. J. Path., 23, 245.

Scott, R. W., and Garvin, C. F. (1941). Amer. Heart J., 22, 56.

Spain, D. M., and Handler, B. J. (1946). Arch. intern. Med., $77,37$.

Welch, K. J., and Kinney, T. D. (1948). Amer. J. Path., $24,729$. 